\title{
Análise dos Itens da Escala de Aconselhamento Profissional (EAP) pelo Modelo de Rasch
}

\author{
Dario Cecilio Fernandes \\ Ana Paula Porto Noronha* \\ Rodolfo A. M. Ambiel \\ Universidade São Francisco, Itatiba, SP, Brasil
}

\begin{abstract}
RESUMO
Esta pesquisa teve como objetivo analisar os itens da Escala de Aconselhamento Profissional segundo o modelo de Rasch, enfocando principalmente o funcionamento diferencial dos itens em relação ao tipo de escola (particular ou pública). Participaram 615 estudantes do ensino médio (68\% do sexo feminino) de instituições do interior do estado de São Paulo, sendo 179 de escola privada e 436 de escola pública, com idades variando entre 14 a 27 anos e média de 16,36. Os resultados mostraram que os itens tiveram, em geral, valores adequados de ajuste. Em relação à análise de DIF, observou-se que 20 itens estavam enviesados, sendo que o favorecimento ao tipo de escola mostrou-se relativamente equilibrado. Sugere-se uma análise mais aprofundada dos itens, para verificar se é necessária a separação da escala em duas versões, ou até mesmo se esse viés é parte do constructo.
\end{abstract}

Palavras-chave: orientação profissional; avaliação psicológica; testes psicológicos.

\begin{abstract}
Analysis of Items of Escala de Aconselhamento Profissional by the Rasch Model

This research aimed to analyze the items of Escala de Aconselhamento Profissional according to Rasch model, foccusing mainly the differential item functioning in relation to type of school (private or public). Were participants 615 high school students (68\% women) from institutions in the state of São Paulo, with 179 private school and 436 public school, with ages ranging from 14 to 27 years and mean of 16,36. Results showed that items had adequate fit values, in general. The analysis of differential item functioning showed that 20 items were biased, but fostering was relatively balanced. It is suggested further analysis of the items to see if it is necessary to separate the scale in two versions, or even if this bias is part of the construct.
\end{abstract}

Keywords: career guidance; psychological evaluation; psychological tests.

O desenvolvimento vocacional pode ser caracterizado como um processo que ocorre ao longo da vida do indivíduo (Osipow, 1999; Savickas, 1995; Super \& Bohn Jr., 1976). Decisões, mudanças e dúvidas vocacionais aparecem não somente no momento da adolescência, sendo cada vez maior o número de pessoas que buscam auxílio e que se encontram em etapas distintas da vida (Prenh, 1997; Teixeira, 1998). Com isso, Osipow (1999) aponta que a orientação profissional (OP) originalmente estava focada na questão da escolha de adolescentes, no entanto, posteriormente passou a englobar um espectro maior de pessoas em razão da crescente frequência de eventos que exigem que elas reavaliem suas decisões de carreira ao longo do tempo.

Outros pesquisadores também observaram que a intervenção em OP deve revestir-se de um caráter educacional que vise preparar as pessoas para as várias mudanças profissionais que tendem a ocorrer ao longo de toda a vida (Guichard, 1993; Guichard \& Huteau, 2001). De acordo com os autores, esta mudança de paradigma vem ocorrendo desde a segunda

* Endereço para correspondência: Ana Paula Porto Noronha - ana.noronha8@gmail.com 
metade do século passado. No Brasil, no entanto, tal compreensão de que ganhou destaque mais acentuado apenas nas duas últimas décadas. A este respeito, Soares (2002) afirma que para o jovem brasileiro, a passagem pelo ensino superior é uma forte expectativa, especialmente daqueles advindos das classes média e alta, o que em alguma medida, reafirma as práticas vigentes dos orientadores profissionais.

Pode-se considerar que a avaliação de interesses profissionais tem sido uma prática constante dos profissionais que realizam a OP em todo o mundo. Contudo, embora o início do estudo sobre interesses tenha se dado há quase oito décadas, com Fryer e sua obra The Measurement of Interests em 1931, pouco se avançou em relação à definição do construto. $\mathrm{O}$ autor definiu interesses como uma relação de aceitaçãorejeição e prazer-desprazer das pessoas com determinados objetos da realidade. Desse modo, o interesse poderia ser compreendido como gostos, ou seja, experiências acompanhadas de sentimentos de satisfação; ou não-gostos, aversões e sentimentos desagradáveis quanto a determinadas atividades profissionais. Em relação à pequena produção conceitual a respeito dos interesses profissionais, Leitão e Miguel (2004) esclarecem que as diferentes abordagens têm enfocado mais a operacionalização do construto que a investigação sobre seu desenvolvimento.

Em produção anterior, os autores (Leitão \& Miguel, 2001) sinalizaram que um dos trabalhos importantes sobre interesses é o de Savickas (1995), no qual o autor afirma que interesses profissionais funcionam como um componente de intermediação do indivíduo com o ambiente. Uma vez assumida essa perspectiva, o autor avalia que necessidades e valores estariam na base dos interesses, que poderiam, então, ser compreendidos como padrões de aceitação de determinados estímulos relacionados a atividades profissionais.

Outros pesquisadores também estudaram os interesses e forneceram teorias buscando estudar sua origem e desenvolvimento. Lent, Brown e Hackett (1994) autores da Teoria Sociocognitiva para o Desenvolvimento de Carreira, cuja elaboração se deu com base nos preceitos da Teoria Sociocognitiva de Bandura (1986), definiram interesses profissionais como padrões de gosto, aversão ou indiferença. Para eles, as crenças de autoeficácia e as expectativas de resultado são anteriores à formação dos interesses, e podem ser entendidos como preditores dos últimos.
No contexto da OP brasileira, a utilização de inventários de interesses tem sido uma prática comum, porém, Noronha, Freitas e Ottati (2003) apresentaram um trabalho que analisou os parâmetros psicométricos de instrumentos de avaliação de interesses utilizados em processos de orientação profissional, de acordo com os relatos dos manuais dos testes. Na ocasião, foram consultados oito instrumentos, avaliando-se alguns aspectos, como autor, editor, ano de publicação, padronização, validade e precisão. Os resultados indicaram a ausência de informações sobre o autor e data de publicação do manual, além de ausência de informações pertinentes aos estudos de padronização, validade e precisão.

Entretanto, mais recentemente tem se observado um crescimento nas pesquisas com testes psicológicos de forma geral (Barroso, 2010) e, em especial, com os de interesses (Ambiel \& Polli, 2011; Noronha \& Ambiel, 2006). Um dos instrumentos pesquisados é a Escala de Aconselhamento Profissional (EAP - Noronha, Sisto \& Santos, 2007), que tem parecer favorável do SATEPSI para uso profissional. A EAP avalia os interesses profissionais, considerando-os como padrões de aceitação, rejeição ou indiferença a determinadas atividades profissionais.

Os estudos a respeito dos interesses profissionais têm sido realizados em vários países e se tem observado que há algumas condições que parecem ter relação com os padrões de preferência, especialmente o gênero (Nunes, Noronha, Godoy, Barros, \& Alves, 2010; Weisgram, Bigler, \& Liben, 2010). Uma outra variável que tem recebido atenção é o nível socioeconômico familiar e os estudos têm mostrado que tal condição parece influenciar as aspirações, valores e interesses profissionais (Alika \& Egbochuku, 2009; Martins \& Noronha, 2010; Noronha, Ambiel, Frigatto, \& Toledo, 2010; Noronha \& Ottati, 2010; Osa-Edoh \& Alutu, 2011).

No Brasil, o nível socioeconômico das famílias pode estar refletido no sistema educacional, uma vez que, de forma geral, há diferenças estruturais, de qualidade do ensino e do desempenho dos alunos entre escolas públicas e particulares (Akkari, 2001). Considerando que a Lei de Diretrizes e Bases da educação brasileira (Brasil, 1996) o ensino médio é definido como o final de um processo de escolarização que tem como objetivo a preparação da pessoa para o trabalho, para a cidadania e para a compreensão dos fundamen- 
tos científico-tecnológicos dos processos produtivos. Sob esta perspectiva, as escolas deveriam favorecer o desenvolvimento do interesse dos estudantes pelas atividades de trabalho, visando uma continuidade dos estudos ou inserção no mercado. Compreendendo que a orientação profissional é uma atividade na qual os psicólogos buscam auxiliar os jovens a escolher uma profissão e que a avaliação dos interesses nesse contexto é importante, este trabalho busca, de forma geral, avaliar as qualidades psicométricas da EAP por meio do modelo de Rasch e, especificamente, verificar se há itens que sejam mais prováveis de serem endossados por estudantes de escolas públicas ou particulares.

Nesse sentido, verificou-se que a literatura brasileira sobre orientação profissional conta com alguns estudos que pesquisaram diferenças entre estudantes de escolas públicas e particulares em variáveis implicadas no processo de escolha profissional. De maneira geral, os resultados sugerem que os de escola pública tendem a privilegiar o aspecto econômico, enquanto que os de escola particular parecem ser mais maduros e acreditar mais que podem se engajar em tarefas relativas à escolha profissional (Noronha \& Ambiel, 2009; Balbinotti, Wiethaeuper \& Barbosa, 2004; 2010; Neiva, Silva, Miranda, \& Esteves, 2005; Primi et al., 2000).

Especificamente a respeito dos interesses profissionais, os artigos encontrados utilizaram análises estatísticas para a comparação entre os grupos (i.e. teste $\mathrm{t}$ e ANOVA) e não para análise dos itens enquanto sua probabilidade de endosso (i.e., DIF), conforme o objetivo deste trabalho. Ainda assim, os estudos serão relatados a seguir.

Estudando a autoeficácia e interesses profissionais, Nunes e Noronha (2009) verificaram em 333 alunos do ensino médio, com média de idade de 15,9 anos se havia diferenças de média em função do sexo, tipo escola e série escolar. Foram utilizados dois instrumentos, a Escala de Autoeficácia para Atividades Ocupacionais (EAAOC) e o Questionário de Busca Autodirigida (SDS). Os resultados mostraram diferenças significativas em função do sexo para os interesses e a autoeficácia. Ainda, verificou-se que a fonte de autoeficácia relativa às experiências pessoais diretas, avaliada pela EAAOC, se diferençou em relação a tipo de escola, sendo a média mais alta para a escola pública. Ressaltou-se que não foram encontradas diferenças entre os padrões de resposta de estudantes de escolas públicas e particulares quanto aos interesses.
Baptista, Noronha e Cardoso (2010) estudaram uma amostra de 85 alunos, com idades entre 14 e 19 anos, provindos de duas escolas, uma pública e outra particular, que responderam a dois instrumentos, quais sejam, a EAP e o Inventário de Percepção de Suporte Laboral (IPSF). Os resultados indicaram algumas correlações significativas e baixas, com destaque para a relação entre interesses em Ciências Biológicas e da Saúde e Ciências Humanas e Sociais Aplicadas com o fator Afetivo-consistente, do IPSF. Com relação a análises de diferença de média, homens tiveram médias maiores nos fatores Adaptação Familiar, do IPSF, e Ciências Agrárias e Ambientais, da EAP. Com relação ao tipo de escola, a única diferença significativa encontrada foi no fator Entretenimento, com maiores médias para os estudantes de escola pública.

Os interesses profissionais foram objeto de estudo de Noronha, Otatti, Mansão e Cézar (2011). As autoras utilizaram a Escala de Aconselhamento Profissional (EAP) em 455 estudantes dos cursos de Engenharias (Ambiental, Elétrica, Industrial, Mecânica e de Produção), Mecatrônica, Ciência da Computação, Arquitetura e Economia, de instituições de ensino superior do interior do estado de São Paulo. Do total de participantes, $63,5 \%$ eram homens e $23,7 \%$ mulheres, com idades entre 17 a 54 anos. O maior interesse de estudantes de Mecatrônica e das Engenharias foram Ciências Exatas e Ciências Agrárias e Ambientais. Artes e Comunicação e Atividades Burocráticas foram as dimensões preferidas pelos alunos de Economia, enquanto os de Arquitetura tiveram maior preferência pelas atividades de Artes e Comunicação. As autoras concluíram que no geral houve coerência entre os cursos e as dimensões preferidas pelos estudantes.

A comparação dos resultados de alunos de ensino médio na EAP com a amostra normativa, composta por estudantes universitários, foi realizada por Noronha, Mansão e Nunes (2012). Para tanto, dois conjuntos de dados foram utilizados, quais sejam, o primeiro composto por 762 estudantes universitários, e o segundo, por 950 alunos do ensino médio. Os achados revelaram que a estrutura fatorial previamente definida para estudantes universitários se repetiu na nova amostra.

Assim, tendo realizado uma breve exposição sobre a situação da área no Brasil, esta pesquisa teve como objetivo verificar o ajuste dos itens da EAP segundo o modelo de Rasch avaliar o funcionamento diferencial dos itens em função de escolas públicas e particulares. 


\section{MÉTODO}

\section{Participantes}

Participaram 615 estudantes do ensino médio, sendo $29,1 \%$ (179) de escolas privadas e $70,9 \%$ (436) de públicas, todas do interior do estado de São Paulo. Desses, $32 \%$ eram do sexo masculino e $68 \%$ do sexo feminino, com idade variando de 14 a 27 anos ( $M_{\text {idade }}$ $=16,36, D P=1,15)$.

\section{Instrumento}

Escala de Aconselhamento Profissional - EAP (Noronha, Sisto, \& Santos, 2007)

A escala é composta por 61 itens, em formato $\mathrm{Li}$ kert de cinco pontos, contendo diversas atividades profissionais. No processo de construção, os autores partiram do princípio de que as pessoas podem gostar de realizar atividades além daquelas típicas de sua ocupação ou profissão. A finalidade do instrumento é fornecer um perfil profissional, caracterizando níveis e intensidades de preferência por determinados grupos de atividades. A escala é aplicada em grupo ou individualmente e não há controle de tempo.

Com o propósito de avaliar as propriedades psicométricas do EAP, os autores do instrumento realizaram estudos de validade e precisão, cuja amostra foi composta por 762 estudantes $(59 \%$ mulheres; Midade =24,1 anos), de 13 diferentes cursos universitários de Minas Gerais e São Paulo. Com relação às evidências de validade, foram realizados estudos de critério e de estrutura interna, chegando-se aos sete fatores propostos, que demonstraram bons níveis de precisão, com coeficientes Alfa de Cronbach variaram entre 0,75 e 0,91 .

A EAP está organizada em sete dimensões, denominadas Ciências Exatas (14 itens), Artes e Comunicação (14 itens), Ciências Biológicas e da Saúde (9 itens), Ciências Agrárias e Ambientais (13 itens), Atividades Burocráticas (13 itens), Ciências Humanas e Sociais Aplicadas (10 itens) e Entretenimento (seis itens). Devido à natureza dos itens, alguns deles apresentaram cargas fatoriais aceitáveis $(>0,30)$ em mais de um fator.

\section{Procedimento}

O projeto foi aprovado pelo Comitê de Ética da Universidade São Francisco. Em seguida, após contato com as escolas, os alunos foram contatados e foram enviados Termos de Consentimento Livre e Esclarecido (TCLE) aos seus pais. Apenas responderam à EAP os alunos cujos responsáveis autorizaram a participação na pesquisa, no caso dos menores de idade. Para os maiores, eles próprios assinaram o TCLE. A aplicação foi realizada em grupo, em salas de aulas cedidas pelas escolas, e durou em média 20 minutos.

\section{Análise de dados}

Os programas estatísticos usados para fazer as análises foram o Winsteps e o SPSS 11.0. Inicialmente, foram extraídas as estatísticas descritivas da amostra. Em seguida foram analisados os índices de adequação dos itens e das pessoas pelo modelo de Rasch, por meio de infit e outfit. Por fim, foi verificado o funcionamento diferencial dos itens (DIF, da sigla em inglês) em relação ao tipo de escola.

A seguir, são explicitados os critérios levados em conta nas análises. Para uma escala ser considerada ajustada ao Modelo de Rasch, Bond e Fox (2001) apontam que o infit e o outfit devem apresentar valores próximos a 1,00 , sendo que os valores podem variar idealmente de 0,70-1,30, sendo aceitáveis valores entre 0,50-1,50 (Linacre, 2002). Além disso, será considerado que um item apresenta DIF, ou seja, que está privilegiando algum dos grupos em relação à probabilidade de concordar com ele, quando os valores de $t$ (de Student) forem superiores 2,0 (Draba, 1977).

\section{RESULTADOS}

Os dados dos itens da EAP foram divididos em suas dimensões e submetidos ao modelo de Rasch para verificar sua adequação ao modelo separadamente. A precisão das dimensões está na Tabela 1. 
Tabela 1.

Coeficientes de precisão obtidos pelo modelo Rasch

\begin{tabular}{lcc}
\multicolumn{1}{c}{ Dimensão } & \multicolumn{2}{c}{ Precisão } \\
& Itens & Pessoas \\
\hline Ciências Exatas (D1) & 0,97 & 0,79 \\
\hline Artes e comunicação (D2) & 0,95 & 0,80 \\
Ciências Biológicas e da Saúde (D3) & 0,98 & 0,81 \\
\hline Ciências Agrárias e Ambientais (D4) & 0,99 & 0,85 \\
\hline Atividades Burocráticas (D5) & 0,98 & 0,79 \\
\hline Ciências Humanas e Sociais Aplicadas (D6) & 0,96 & 0,70 \\
\hline Entretenimento (D7) & 0,97 & 0,69 \\
\hline
\end{tabular}

Observa-se que o maior índice de precisão foi de 0,99 para os itens e 0,85 para as pessoas, com mínimo de 0,95 e 0,69 respectivamente, indicando boa preci- são. Os parâmetros de ajustes dos itens e pessoas estão dispostos na Tabela 2.

Tabela 2.

Parâmetros de Ajuste de Itens e Pessoas, e precisão pelo Modelo Rasch, por dimensão

\begin{tabular}{|c|c|c|c|c|c|c|c|c|c|}
\hline \multirow{2}{*}{ Dimensão } & \multirow{2}{*}{ Parâmetros } & \multicolumn{4}{|c|}{ Itens } & \multicolumn{4}{|c|}{ Pessoas } \\
\hline & & Dificuldade & Infit & Outfit & Erro & Habilidade & Infit & Outfit & Erro \\
\hline \multirow{4}{*}{ D 1} & Média & 0,00 & 1,01 & 1,02 & 0,04 & $-0,65$ & 1,04 & 1,02 & 0,30 \\
\hline & $\mathrm{Dp}$ & 0,23 & 0,17 & 0,27 & 0,00 & 0,79 & 0,52 & 0,53 & 0,13 \\
\hline & Máxima & 0,35 & 1,45 & 1,81 & 0,04 & 3,07 & 2,96 & 3,90 & 0,99 \\
\hline & Mínima & $-0,38$ & 0,75 & 0,72 & 0,04 & $-2,91$ & 0,10 & 0,10 & 0,21 \\
\hline \multirow{4}{*}{ D 2} & Média & 0,00 & 1,01 & 1,04 & 0,04 & $-0,39$ & 1,05 & 1,04 & 0,27 \\
\hline & $\mathrm{Dp}$ & 0,17 & 0,19 & 0,30 & 0,00 & 0,72 & 0,48 & 0,48 & 0,10 \\
\hline & Máxima & 0,26 & 1,47 & 1,88 & 0,04 & 2,85 & 2,94 & 3,12 & 0,98 \\
\hline & Mínima & $-0,29$ & 0,70 & 0,65 & 0,03 & $-2,77$ & 0,05 & 0,06 & 0,20 \\
\hline \multirow{4}{*}{ D 3} & Média & 0,00 & 1,01 & 1,01 & 0,04 & $-0,19$ & 1,01 & 1,01 & 0,36 \\
\hline & $\mathrm{Dp}$ & 0,28 & 0,12 & 0,15 & 0,00 & 0,98 & 0,55 & 0,61 & 0,15 \\
\hline & Máxima & 0,33 & 1,13 & 1,17 & 0,04 & 2,77 & 3,33 & 4,38 & 0,99 \\
\hline & Mínima & $-0,52$ & 0,74 & 0,69 & 0,04 & $-2,62$ & 0,10 & 0,10 & 0,27 \\
\hline \multirow{4}{*}{ D 4} & Média & 0,00 & 1,01 & 1,06 & 0,04 & $-0,14$ & 1,04 & 1,06 & 0,30 \\
\hline & $\mathrm{Dp}$ & 0,37 & 0,22 & 0,27 & 0,00 & 0,90 & 0,55 & 0,66 & 0,09 \\
\hline & Máxima & 0,75 & 1,46 & 1,64 & 0,04 & 3,47 & 4,02 & 8,77 & 1,00 \\
\hline & Mínima & $-0,46$ & 0,72 & 0,71 & 0,04 & $-2,76$ & 0,00 & 0,00 & 0,25 \\
\hline \multirow{4}{*}{ D 5} & Média & 0,00 & 1,01 & 1,03 & 0,04 & $-0,35$ & 1,04 & 1,03 & 0,27 \\
\hline & $\mathrm{Dp}$ & 0,24 & 0,18 & 0,25 & 0,00 & 0,70 & 0,53 & 0,51 & 0,09 \\
\hline & Máxima & 0,41 & 1,50 & 1,74 & 0,04 & 2,10 & 3,02 & 2,91 & 0,98 \\
\hline & Mínima & $-0,38$ & 0,82 & 0,80 & 0,04 & $-3,02$ & 0,09 & 0,10 & 0,22 \\
\hline \multirow{4}{*}{ D 6} & Média & 0,00 & 1,01 & 1,03 & 0,04 & $-0,32$ & 1,03 & 1,02 & 0,30 \\
\hline & $\mathrm{Dp}$ & 0,19 & 0,13 & 0,15 & 0,00 & 0,63 & 0,51 & 0,53 & 0,09 \\
\hline & Máxima & 0,35 & 1,23 & 1,25 & 0,04 & 2,79 & 3,30 & 4,60 & 0,99 \\
\hline & Mínima & $-0,32$ & 0,82 & 0,82 & 0,03 & $-2,72$ & 0,00 & 0,00 & 0,25 \\
\hline \multirow{4}{*}{ D 7} & Média & 0,00 & 1,01 & 1,02 & 0,04 & $-0,29$ & 1,01 & 1,01 & 0,44 \\
\hline & $\mathrm{Dp}$ & 0,23 & 0,25 & 0,30 & 0,00 & 0,93 & 0,63 & 0,66 & 0,16 \\
\hline & Máxima & 0,26 & 1,42 & 1,43 & 0,04 & 2,41 & 3,33 & 4,03 & 0,98 \\
\hline & Mínima & $-0,35$ & 0,72 & 0,68 & 0,04 & $-2,35$ & 0,05 & 0,05 & 0,33 \\
\hline
\end{tabular}

Em relação aos itens, a média de infit foi de 1,01 para todas as dimensões e quanto ao outfit variou de 1,02 a 1,06, indicativo de que, no geral, a maior parte deles foi respondida dentro do padrão esperado $(1,00)$ conforme o modelo de Rasch (Bond \& Fox, 2001). Analisando esses dados mais detidamente, verificou- 
se que os valores de infit estiveram no intervalo de 0,70 a 1,50, sugerindo que nem todos os itens enquadraram-se no parâmetro 0,70-1,30, característico de bom ajuste. Mais detalhadamente, cinco itens com desajustes apresentaram valores de infit superiores a 1,30 , mas nenhum caso foi superior a 1,50 , valor considerado por Linacre (2002) como o limite máximo para se aceitar um item. Todavia, de modo geral, a variabilidade de infit esteve dentro dos níveis esperados.

Por sua vez, a média de outfit encontrada variou de 1,02 a 1,06, o que aponta para uma boa adequação dos itens ao modelo de Rasch. Entretanto, a variação de outfit esteve no intervalo de 0,65 a 1,88 , indicando que houve itens fora do intervalo esperado. Mais especificamente, ficou evidenciado que quatro itens apresentaram valores acima de 1,50 que pertenciam as dimensões Ciências Exatas, Artes e comunicação e Ciências Agrárias e Ambientais; quatro acima de 1,30 das dimensões Artes e comunicação, Ciências Agrárias e Ambientais, e Entretenimento; dois abaixo de
0,70 das dimensões Artes e comunicação e Entretenimento. Vale destacar que índices de outfit acima de 1,50 são considerados inadequados.

No que se refere às pessoas, evidenciou-se que a maioria apresentou o padrão esperado $(1,00)$ em ambos os parâmetros de ajuste, uma vez que a média de infit variou de 1,01 a 1,05 e a de outfit de 1,01 a 1,06. Observou-se que o intervalo de variação do outfit $(0,00-8,77)$ foi maior que o de infit $(0,00-3,33)$.

Outro aspecto analisado no presente estudo referese à análise da variação da dificuldade dos itens da escala, que foi de $-0,52$ a 0,75 , ficando entre 1 e -1 logit, intervalo que avalia as pessoas com maior precisão e menos erros. A média foi 0,00 para todas as dimensões, indicativo de que os itens se mostraram com dificuldade média. Em relação ao funcionamento diferencial dos itens (DIF), a Tabela 3 resume os resultados encontrados na análise de diferenciação entre tipos de escola.

Tabela 3.

\begin{tabular}{|c|c|c|c|c|c|c|c|}
\hline Escola & DIF & Escola & DIF & DIF Contraste & $\mathbf{t}$ & Welch & Itens \\
\hline pública & $-0,13$ & particular & $-0,48$ & 0,35 & 4,24 & 0,0000 & eap_27 \\
\hline pública & 0,33 & particular & 0,11 & 0,22 & 2,40 & 0,0169 & eap_38 \\
\hline pública & $-0,29$ & particular & 0,08 & $-0,37$ & $-4,34$ & 0,0000 & eap_41 \\
\hline pública & 0,13 & particular & $-0,13$ & 0,26 & 3,04 & 0,0025 & eap_42 \\
\hline pública & $-0,29$ & particular & $-0,11$ & $-0,18$ & $-2,13$ & 0,0337 & eap_46 \\
\hline pública & $-0,05$ & particular & 0,47 & $-0,52$ & $-5,54$ & 0,0000 & eap_60 \\
\hline pública & $-0,34$ & particular & $-0,19$ & $-0,15$ & $-2,02$ & 0,0443 & eap_5 \\
\hline pública & $-0,14$ & particular & 0,04 & $-0,18$ & $-2,36$ & 0,0190 & eap_6 \\
\hline pública & $-0,05$ & particular & $-0,21$ & 0,15 & 2,02 & 0,0438 & eap_7 \\
\hline pública & $-0,25$ & particular & $-0,09$ & $-0,16$ & $-2,11$ & 0,0357 & eap_34 \\
\hline pública & 0,38 & particular & 0,18 & 0,20 & 2,25 & 0,0249 & eap_18 \\
\hline pública & 0,60 & particular & 0,28 & 0,31 & 3,43 & 0,0007 & eap_13 \\
\hline pública & $-0,38$ & particular & $-0,13$ & $-0,25$ & $-2,78$ & 0,0057 & eap_17 \\
\hline pública & 0,08 & particular & $-0,23$ & 0,31 & 3,50 & 0,0005 & eap_20 \\
\hline pública & 0,69 & particular & 0,93 & $-0,24$ & $-2,34$ & 0,0197 & eap_60 \\
\hline pública & $-0,48$ & particular & $-0,13$ & $-0,35$ & $-4,41$ & 0,0000 & eap_3 \\
\hline pública & $-0,04$ & particular & $-0,24$ & 0,19 & 2,53 & 0,0060 & eap_23 \\
\hline pública & 0,33 & particular & 0,14 & 0,19 & 2,34 & 0,0197 & eap_43 \\
\hline pública & $-0,17$ & particular & 0,05 & $-0,23$ & $-2,91$ & 0,0038 & eap_59 \\
\hline pública & $-0,06$ & particular & 0,38 & $-0,44$ & $-5,18$ & 0,0000 & eap_61 \\
\hline
\end{tabular}


Em relação ao estudo do funcionamento diferencial dos itens, $20(32,79 \%)$ itens no total apontaram a presença de DIF, sendo seis para primeira dimensão (Ciências Exatas), quatro para segunda (Artes e comunicação), uma para terceira (Ciências Biológicas e da Saúde), cinco para quarta (Ciências Agrárias e Ambientais), uma para quinta (Atividades burocráticas), três para sexta (Ciências humanas e sociais aplicadas), e nenhuma para sétima (Entretenimento). Além disso apenas um item se repetiu com DIF, sendo ele o número 60, pertencente às dimensões $1 \mathrm{e} 4$.

A porcentagem encontrada pode ser considerada alta e que deve ser levada em conta quando da interpretação dos resultados da EAP. Este resultado mostra que certos itens têm maior probabilidade de serem endossados por estudantes de um dos tipos de escola e é evidenciado nas colunas de DIF na Tabela 3, sendo que os valores menores indicam qual tipo está sendo privilegiado. Dessa forma, verifica-se que em nove itens, o favorecimento foi para os estudantes de escolas particulares (itens, eap_27, eap_38, eap_42, eap_7, eap_18, eap_13, eap_20, eap_23, eap_43) e em 11 itens, o benefício foi para estudantes do sistema público (itens - eap_41, eap_46, eap_60, eap_5, eap_6, eap_34, eap_17d3d4, eap_60, eap_3, eap_59, eap_61).

Aprofundando essa análise, verifica-se que os itens que favoreceram os estudantes de instituições particulares estavam relacionados principalmente às dimensões Ciência Biológica e da Saúde e Ciências Agrárias e Ambientais e, em menor número, Ciências Humanas e Sociais Aplicadas. Por outro lado, os itens favoráveis aos estudantes de escolas públicas foram predominantemente das dimensões Artes e Comunicação e Ciências Exatas.

\section{DISCUSSÃO E CONCLUSÕES}

Esta pesquisa teve como finalidade analisar os itens da EAP pelo modelo de Rasch e verificar as possíveis diferenças no funcionamento dos itens que avaliam a preferência por atividades profissionais entre estudantes do ensino médio de escolas particulares e públicas. Há que se considerar que na literatura brasileira não foram encontrados artigos publicados que tivessem utilizado análises de Rasch, com base na Teoria de Resposta ao Item (TRI) de instrumentos de avaliação de interesses.
Os resultados deste estudo apontaram que, de forma geral, os itens da EAP são adequados no que toca aos seus índices de ajuste, não tendo sido encontrados resultados que possam ser considerados problemáticos, uma vez que apenas um item extrapolou o valor máximo de outfit. Isso significa dizer que em um item houve algum padrão de resposta inesperado, no sentido de ter sido endossado por alguém cujo nível do traço latente não é compatível com o item endossado (Bond \& Fox, 2001).

Em relação ao funcionamento diferencial, verificou-se a existência de itens que favoreceram tanto as escolas particulares quanto públicas. Os itens que beneficiam os estudantes das instituições particulares estão relacionados a área da saúde, ciências biológicas, ciências agrárias, ambientais, ciências humanas e sociais aplicadas, enquanto que os itens que favorecem as escolas públicas estão ligados a artes e comunicações e ciências exatas. Tal resultado sugere que os estudantes de escolas particulares tendem a pontuar mais em itens de dimensões mais diversificadas, uma vez que os nove itens com DIF eram de seis dimensões diferentes, enquanto que os 11 que apresentaram resultados favorecendo estudantes de escolas públicas distribuíram-se em apenas duas dimensões.

Considerando pesquisas anteriores que mostraram que o nível socioeconômico familiar está relacionado com a diversificação de experiências relacionadas ao desenvolvimento vocacional (Alika \& Egbochuku, 2009; Martins \& Noronha, 2010; Noronha \& Ottati, 2010; Osa-Edoh \& Alutu, 2011), o resultado desta pesquisa parece ir na mesma direção, uma vez que o favorecimento em itens de dimensões de interesses diversas podem sugerir um maior contato de estudantes de escolas particulares com conteúdos relacionados ao mundo do trabalho. Por outro lado, tal resultado parece não contemplar o que diz a Lei de Diretrizes e Bases (Brasil, 1996), que prega que o ensino médio deveria favorecer o pleno desenvolvimento de interesses e a intenção de continuidade dos estudos para todos, independentemente da categoria da escola. Ainda, é importante destacar que os resultados diferem de outros estudos encontrados na literatura, em que pese a diferença na metodologia de análise de dados (Baptista et al., 2010; Nunes \& Noronha, 2009)

Tendo em vista que quase metade dos itens apresentou uma possibilidade de viés, pode-se pensar que haveria diferenças entre as escolhas profissionais de 
estudantes da escola particular e pública. Contudo, os vieses detectados podem ser parte do constructo, o que sugere cautela na análise do conteúdo dos itens (Sisto, 2006). No entanto, há que se ressaltar que alguns autores (Noronha \& Ambiel, 2009; Balbinotti et al., 2004; Neiva et al, 2005; Primi et al, 2000; dentre outros) apontam que alunos da escola pública tendem a privilegiar o aspecto econômico.

Com isso, os objetivos dos trabalhos foram alcançados, haja vista que a escala se adaptou bem ao modelo de Rasch. No que se refere ao DIF verificou-se uma grande quantidade de itens enviesados, algo que deve ser estudado mais profundamente, para buscar explicações para o achado. Portanto, sugerem-se novas pesquisas com amostras diversificadas quanto ao tipo de escola.

\section{REFERÊNCIAS}

Akkari, A. J. (2001). Desigualdades educativas estruturais no Brasil: entre estado, privatização e descentralização. Educação e Sociedade, 22(74), 163-189.

Alika, H. I., \& Egbochuku, E. O. (2009). Vocational Interest, Counselling, Socio-economic Status and Age as Correlates of Re-entry of Girls into School in Edo State. Edo Journal of Counselling, 2(1), 9-16.

Ambiel, R. A. M., \& Polli, M. F. (2011). Análise da produção científica brasileira sobre avaliação psicológica em orientação profissional. Estudos interdisciplinares em psicologia, 2(1), 103-121.

Balbinotti, M. A. A., Wiethaeuper, D., \& Barbosa, M. L. L. (2004). Níveis de cristalização de preferências profissionais em alunos de ensino médio. Revista Brasileira de Orientação Profissional, 5(1), 15-28

Bandura, A. (1986). Social foundations of thought and action: a social cognitive theory. Upper Saddle River, NJ: Prentice-Hall.

Baptista, M. N., Noronha, A. P. P., \& Cardoso, H. F. (2010). Relações entre suporte familiar e interesses profissionais. Revista Salud \& Sociedad, 1(1), 28-40.

Barroso, S. M. (2010). Avaliação psicológica: análise das publicações disponíveis na SciELO e BVS-Psi. Fractal, 22(1), 141-154.

Bond, T. G., \& Fox, C. M. (2001). Applying the Rasch model: Fundamental Measurement in the Human Sciences. Mahawah, New Jersey: Lawrence Erlabum.

Brasil. (1996). Lei n. 9.394, de 20 de dezembro de 1996. Estabelece as diretrizes e bases da educação nacional. Retirado em 06/02/2015, Presidência da República Federativa do Brasil no World Wide Web: http:// www.presidencia.gov.br/
Draba, R. E. (1977). The identification and interpretation of item Bias (Research Memorandum No. 25). Chicago: The University of Chicago, Department of Education, Education Statistics Laboratory. Retirado em: 02/2015, no World Wide Web: http://www.rasch.org/memo25. htm

Guichard, J. (1993). L'école et les représentations d'avenir des adolescents. Paris: PUF, collection: Le Psychologue.

Guichard, J., \& Huteau, M. (2001). Psychologie de l'Orientation. Paris: Dunod.

Leitão, L. M., \& Miguel, J. P. (2001). Os interesses revisitados. Psychologica, 26, 79-104.

Leitão, L. M., \& Miguel, J. P. (2004). Avaliação dos Interesses. Em L. M. Leitão (Org.), Avaliação psicológica em orientação escolar e profissional (pp. 179-262). Coimbra: Quarteto.

Lent, R. W. Brown, S. D., \& Hackett, G. (1994). Towards a unifying social cognitive theory of career and academic interests, choice and performance. Journal of Vocational Behavior, 45(1), 79-122.

Linacre J. M. (2002). What do infit and outfit, meansquared and standardized mean? Rasch Measurement Transactions, 16(2), 878. Acessado em 10 de julho de 2006, do site http://209.238.26.90/rmt/rmt82a.htm.

Martins, D. F., \& Noronha, A. P. P. (2010). Interesse profissional e características socioeconômicas de estudantes do Ensino Médio. Psico, 41, 76-84.

Neiva, K. M. C., Silva, M. B., Miranda, V. R., \& Esteves, C. (2005). Um estudo sobre a maturidade para a escolha profissional de alunos do ensino médio. Revista Brasileira de Orientação Profissional 6(1), 1-14.

Noronha, A. P. P., \& Ottati, F. (2010). Interesses profissionais de jovens e escolaridade dos pais. Revista Brasileira de Orientação Profissional 11(1), 37-47.

Noronha, A. P. P., \& Ambiel, R. A. M. (2006). Orientação profissional e vocacional: análise da produção científica. Psico USF, 11(1), 75-84.

Noronha, A. P. P., \& Ambiel, R. A. M. (2009). Fontes de eficácia e interesses profissionais: relações entre pais e filhos. Evaluar, 8, 32-45.

Noronha, A. P. P., Ambiel, R. A. M., Frigatto, V., \& Toledo, C. C. R. (2010). Relações entre interesses, intenções e critérios de escolha profissional. Estudos Interdisciplinares em Psicologia, 1(1), 2-25.

Noronha, A. P. P., Freitas, F. A., \& Ottati, F. (2003). Análise de instrumentos de avaliação de interesses profissionais. Psicologia: Teoria e Pesquisa, 19(3), 287-291.

Noronha, A. P. P., Mansão, C. S. M., \& Nunes, M. F. O. (2012). Interesses profissionais e Personalidade: análise correlacional a partir do ATPH e BFP. Actualidades en Psicología, 26, 73-86. 
Noronha, A. P. P., Otatti, F., Mansão, C. S. M., \& Cezar, E. O. (2011). Aplicação da Escala de Aconselhamento Profissional em Estudantes Universitários. Acta Colombiana de Psicologia, 14, 155-164.

Noronha, A. P., Sisto, F., \& Santos, A. A. A. (2007). Escala de aconselhamento profissional- EAP- manual técnico. São Paulo: Vetor.

Nunes, M. F. O., \& Noronha, A. P. P. (2009). Auto-eficácia para atividades ocupacionais e interesses profissionais em estudantes do ensino médio. Psicologia: Ciência e Profissão, 29(1), 102-115.

Nunes, M. F. O., Noronha, A. P. P., Godoy, S., Barros, M. V. C., \& Alves, M. H. (2010). Levantamento de Preferências Profissionais com jovens de Ensino Fundamental e Médio. Encontro (Santo André), 13, 45-58.

Osa-Edoh, G. I., \& Alutu, A. N. G. (2011). Parents' socioeconomic status and its effects in students' educational values and vocational choices. European Journal of Educational Studies, 3(1), 11-21.

Osipow, S. H. (1999). Assessing career indecision. Journal of Vocational Behavior, 55, 147- 154.

Prenh, D. R. (1997). Orientação ocupacional: Redirecionando a vida profissional. [Resumos]. Em Anais, III Simpósio Brasileiro de Orientadores Profissionais. Canoas, RS: ABOP.

Primi, R., Munhoz, A. M. H., Bighetti, C. A., Nucci, E. P., Pelegrini, M. K., \& Moggi, M. A. (2000). Desenvolvi- mento de um Inventário de Levantamento das Dificuldades da Decisão Profissional. Psicologia Reflexão e Crítica, 13(3), 451-463.

Savickas, M. L. (1995). Constructivist counseling for career indecision. The Career Development Quarterly,43, 363373.

Sisto, F. F. (2006). O funcionamento diferencial do item. Psico-USF, 11(1), 35-43.

Soares, D. H. P. (2002). Como trabalhar a ansiedade e o estresse frente ao vestibular. Em R. S. Levenfus \& D. H. P. (Orgs.). Orientação vocacional ocupacional: novos achados teóricos, técnicos e instrumentais para a clínica, a escola e a empresa. Porto Alegre: Artmed.

Super, D. E., \& Bohn Jr., M. J. (1976). Psicologia Ocupacional (E. Nascimento \& F. Santos, Trads.). São Paulo: Atlas.

Teixeira, M. A. P. (1998). Mudança profissional: Uma abordagem fenomenológica. Dissertação de Mestrado não publicada. Curso de Pós-Graduação em Psicologia do Desenvolvimento, Universidade Federal do Rio Grande do Sul. Porto Alegre, RS.

Weisgram, E. S., Bigler, R. S., \& Liben, L. S. (2010). Gender, values, and occupational interests among children, adolescents, and adults. Child Development, 81, 778796. 